\title{
Comparison between computed balance velocities and GPS measurements in the Lambert Glacier basin, East Antarctica
}

\author{
Laurent TeStut, ${ }^{1}$ Raghael HURD, ${ }^{2}$ Righard COLEMAN, ${ }^{2,3,4}$ Frédérique RÉmY, \\ BENOÎT LEGRÉSY ${ }^{1}$ \\ ${ }^{1}$ Laboratoire d'Étude en Géophysique et Océanographie Spatiale, 18 avenue Edouard Belin, 31055 Toulouse Cedex, France \\ E-mail: laurent.testut@cnes.fr \\ ${ }^{2}$ Centre for Spatial Information Science, University of Tasmania, Box 252-76, Hobart, Tasmania 7001, Australia \\ ${ }^{3}$ CSIRO Marine Research, Box 1538, Hobart, Tasmania 7001, Australia \\ ${ }^{4}$ Antarctic CRC, Box 252-80, Hobart, Tasmania 7001, Australia
}

\begin{abstract}
Comparisons between computed balance velocities, obtained from two different computing schemes, and global positioning system (GPS)-derived velocities were made in the Lambert Glacier basin region, East Antarctica. The two computing schemes used for the balance-velocity computations (a flowline (FL) scheme (Rémy and Minster, 1993) and a finite-difference (BW) scheme (Budd and Warner, 1996; Fricker and others, 2000) ) were first evaluated and compared. One of the key issues studied was the spatial resolution of the digital elevation model (DEM), representing the surface topography of the ice sheet, and the sensitivity of the balance velocities to the length of smoothing applied to the DEM. Comparison with the GPS velocities validated the two schemes to within 5-25\% but showed the high sensitivity of the flowline method to the length scale of the smoothing. The finite-difference scheme was found to be robust to the chosen smoothing scale, but the balance-velocity values increased when a finer-resolution DEM was used. Both FL and BW computing schemes tended to overestimate the balance velocities in comparison with the GPS values; some of this discrepancy can be attributed to ice-sheet sliding.
\end{abstract}

\section{INTRODUGTION}

Ice sheets play a major role in studies of global climate change and its impact on mean sea level. Antarctica is the largest reservoir of fresh water in the world, and any changes in its volume will therefore have a direct impact on the global mean sea level. To predict future changes of the ice-sheet volume, both the changes in atmospheric conditions and the dynamic response of the ice sheets require further investigation. Although our knowledge of ice physics, glacier dynamics and techniques for monitoring and modelling ice sheets has considerably improved during the last 50 years, many physical processes affecting an ice sheet still remain poorly understood. This lack of understanding is partly due to the intrinsic complexity of the behaviour of the ice but is also caused by the present lack of accurate data with global coverage, especially for the accumulation rates and ice thicknesses.

Over the last few decades, it has been established that the ice discharge from the Antarctic ice sheet is mainly controlled by outlet glaciers and ice streams. A broad description (Drewry and others, 1982) shows that $90 \%$ of the ice of the Antarctic ice sheet is discharged by fast-flow features which occupy only $13 \%$ of the coastline (Morgan and others, 1982). Joughin and others (1999), using synthetic aperture radar (SAR) techniques in the West Antarctic ice sheet region, show that tributaries can provide a spatially extensive transition between slow inland flow and rapid ice-stream flow. More recently, an estimation of balance velocity over the whole continent confirmed this result (Bamber and others, 2000b). This evidence, which shows that ice sheets are controlled by a complex tributary-type flow, has changed our view of the dynamics of an ice sheet.

During the last 10 years, computation of ice-sheet balance velocity has become a useful tool in the study of ice-sheet dynamics. Two main computing methods have been developed: the flowline method, which is the historical method by which the balance velocity is computed (Radok and others, 1982; Rémy and Minster, 1993; Joughin and others, 1997), and a finite-difference scheme developed by Budd and Warner (1996) (Bamber and Huybrechts, 1996; Fricker and others, 2000). The aim of this paper is to evaluate these two methods in an area where a series of high-precision global positioning system (GPS) measurements are available.

\section{GOMPUTING SGHEMES}

\section{Assumptions}

A classic way of estimating the drainage pattern in Antarctica is to compute the so-called "balance velocity". The balance velocity corresponds to the depth-averaged velocity needed at any point to maintain the ice sheet in a state of balance. Two assumptions are required to make the computation. The first one presumes that the ice sheet is in a state of balance, such that the outward flow distribution matches the net accumulation without considering any time vari- 

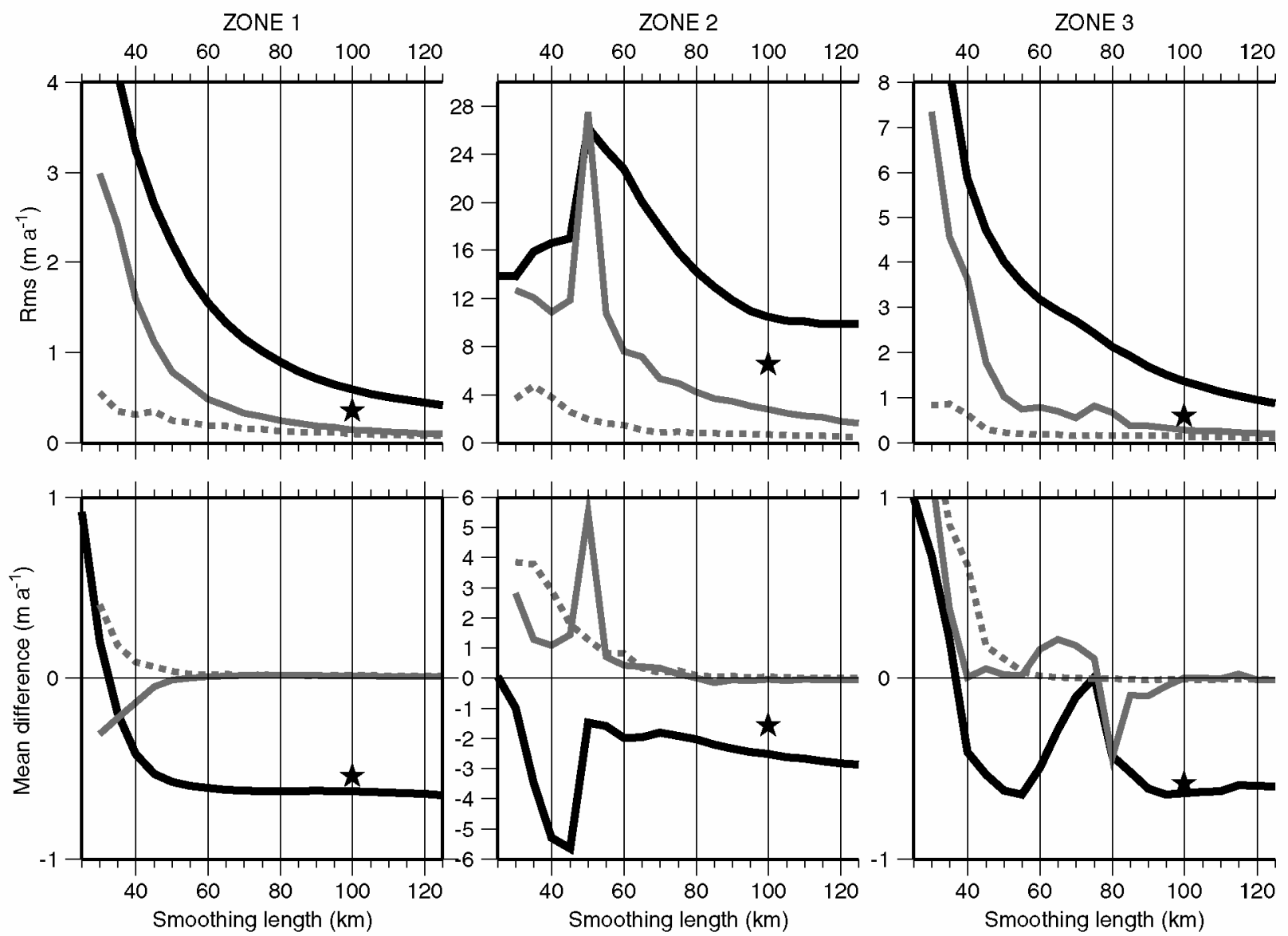

Fig. 1. Mean velocity difference and its rms variation. The solid black line is the difference between the FL balance velocity and the $B W$ balance velocity. The grey lines ( full $=F L$, dashed $=B W$ ) are the differences between computed velocities associated with the smoothing distance $L$ and velocities associated with the smoothing distance ( $L-5 \mathrm{~km})$, and show the convergence of these two methods. The three upper plots represent the rms of these differences computed for the three zones, and the three lower plots give the mean difference (in $\mathrm{m}^{-1}$ ). The star in each box shows the corresponding quantity computed using a $1 \mathrm{~km} D E M$.

ation in the thickness. The second assumption considers that, in a first approximation, the surface slope corresponds to the dominant direction of the ice flow. Under these assumptions, the mass-balance equation of an ice sheet can be written:

$$
U_{\mathrm{b}}(x) H(x) l_{0}=\int_{0}^{x} b(x) l(x) \mathrm{d} x,
$$

where $x$ is the curvilinear distance from the flow source $(x=0), U_{\mathrm{b}}$ is the balance velocity, $H$ is the local thickness, $b(x)$ is the net accumulation rate and $l_{0}$ is the initial distance between two flowlines (we used $l_{0}=5 \mathrm{~km}$ ). Hence, with a given distribution over the ice sheet of net accumulation rate and ice thickness, the balance velocity can be estimated.

\section{Flowline and finite-difference schemes}

The flowline scheme (now called FL) is based on the upstream calculation of the surface between two distinct flowlines, originally lying on a contour on both sides of a point (Rémy and Minster, 1993). The upstream paths of the two flowlines are then computed at $5 \mathrm{~km}$ intervals along the flowlines, with a local slope calculated over a $10 \mathrm{~km}$ length scale. In this way, each point along the flowline is computed separately from the others and is free to evolve through the specified grid resolution.

The finite-difference scheme is based on Fricker and others (2000), which is an improvement of the Budd and Warner
(1996) method (now called BW). This method is based on a two-dimensional, finite-difference scheme, which distributes the accumulation to the eight neighbouring cells according to the slope direction. The computation is carried out for each cell, previously sorted in decreasing order, so that each cell of the grid receives the flux of all of the previous cells.

\section{Input dataset}

Computation of the balance velocities was performed in the Lambert Glacier basin (LGB) region, using the two methodologies discussed above. Two different $5 \mathrm{~km}$ digital elevation models (DEMs) of the Antarctic ice sheet, derived from the altimetric data of the European Remote-sensing Satellite (ERS-1) (Liu and others, 1999; Rémy and others, 1999), were chosen to assess the variability due to topography. The BEDMAP thickness compilation (Lythe and others, 2001) and a recent compilation of accumulation rates (Vaughan and others, 1999) were used as the other data-input sources.

\section{COMPARISON OF THE TWO METHODS}

Few comparisons have been made between the two computation schemes. Bamber and others (2000a) compared the two methods across the northeast Greenland ice stream and noted that the flowline method tends to over-concentrate the flow at the centre of the stream and overestimates 
Smoothing length $=50 \mathrm{~km}$

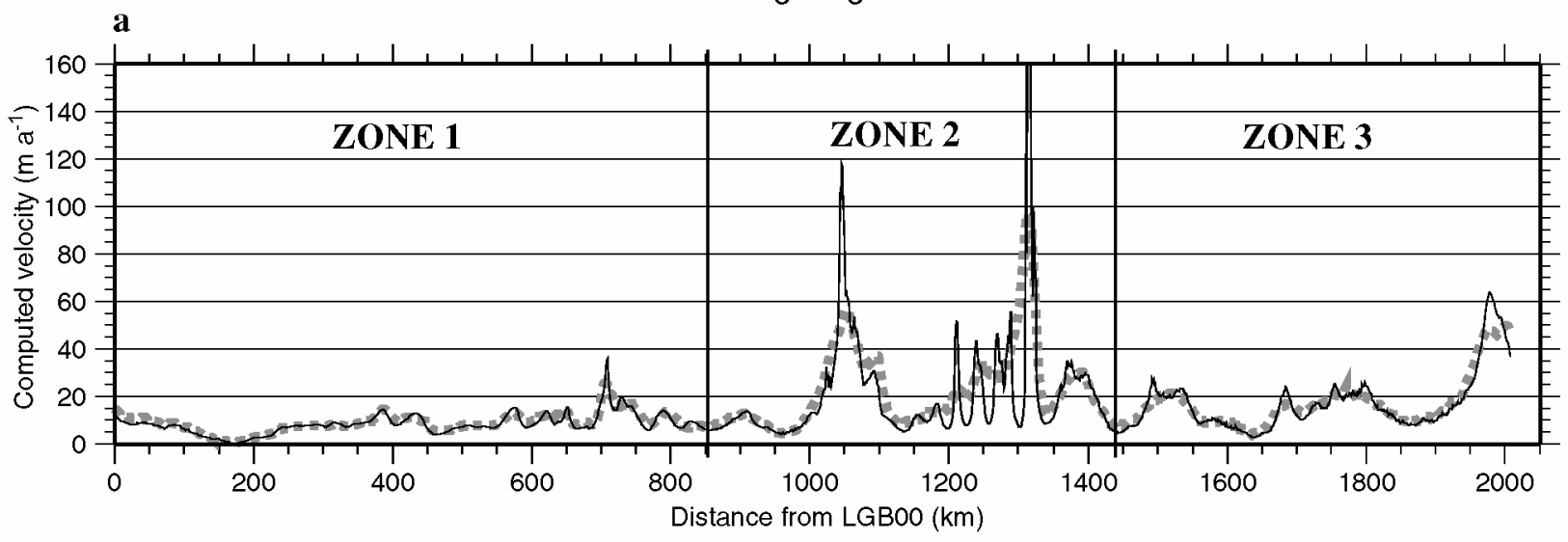

Smoothing length $=100 \mathrm{~km}$

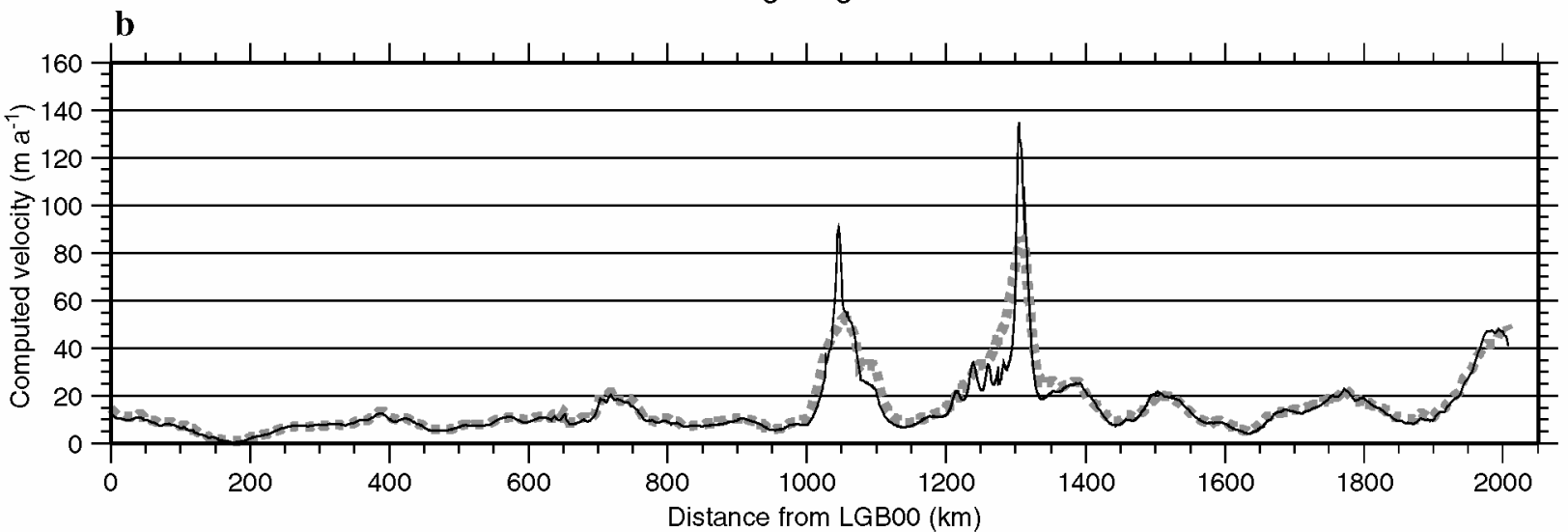

Smoothing length $=100 \mathrm{~km} \&$ Grid size $=1 \mathrm{~km}$

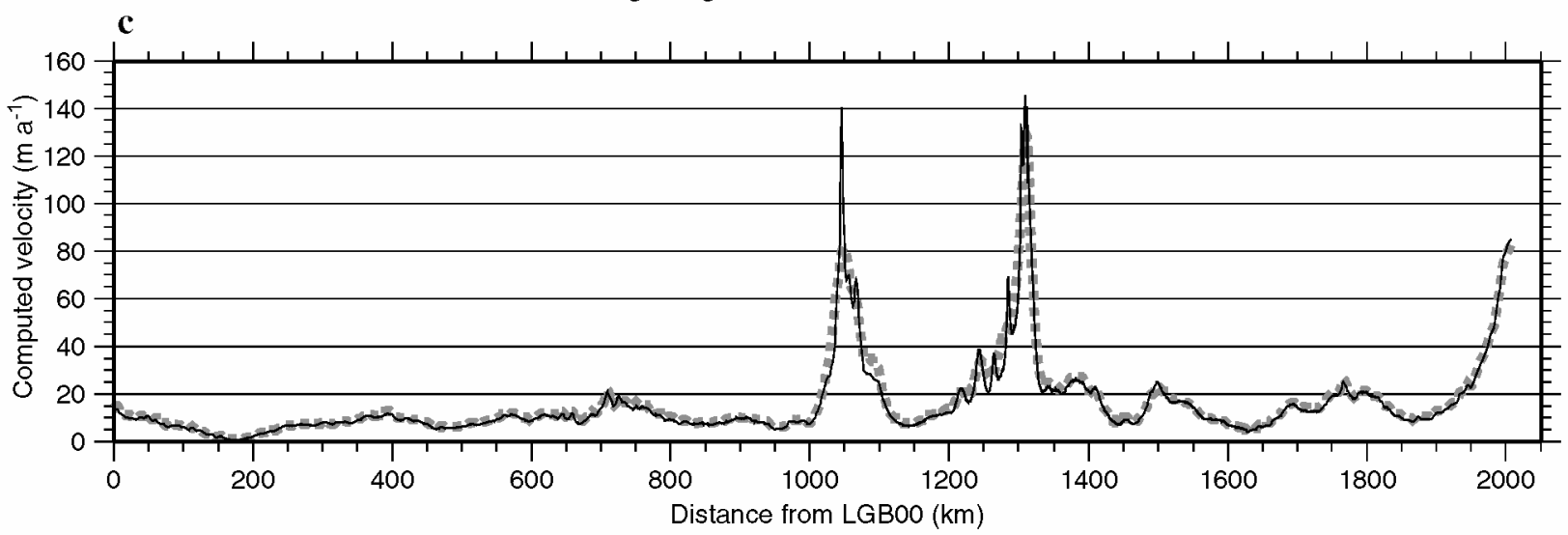

Fig. 2. The three curves show the computed balance velocity for the digitized profile along the LGB traverse route for varying smoothing or grid sizes. Dashed grey lines represent the BW computed velocity, and solid black lines are used for the FL values. (a) and (b) are computed using a DEM grid size of $5 \mathrm{~km}$, and (c) uses a DEM grid size of $1 \mathrm{~km}$.

the balance velocity. This comparison was made with a $20 \mathrm{~km}$ smoothed DEM of the Greenland ice sheet. We will investigate here the role of this smoothing distance in our area of interest and compare against in situ GPS values in section 4 .

\subsection{The role of the smoothing scale}

The question of what spatial smoothing should be applied to various data sources when we undertake any flow computations is problematic. From a methodological point of view, under certain smoothing length scales, neither method is able to produce a coherent picture of the flow because each is sensitive to small-scale undulations in the topography, i.e. the presence of numerous isolated depressions. From a glaciological point of view, as the effect of longitudinal stresses is not included under the assumption of downslope flow, a classical smoothing distance of 10-20 times the ice thickness is applied to the topography in order to remove the effect of longitudinal stresses (Paterson, 1994). However, this length scale is highly dependent on the ice rheology and flow conditions.

The approach adopted here is to study the sensitivity of both schemes to a chosen smoothing length scale applied to the topography. Heights in the Lambert Glacier drainage basin region were extracted from the available DEMs and smoothed with a Gaussian moving-average box of varying size $L$. The DEM was smoothed from 5 to 25 times the gridcell size (i.e. from 25 to $125 \mathrm{~km}$ ), leading to 21 different ver- 


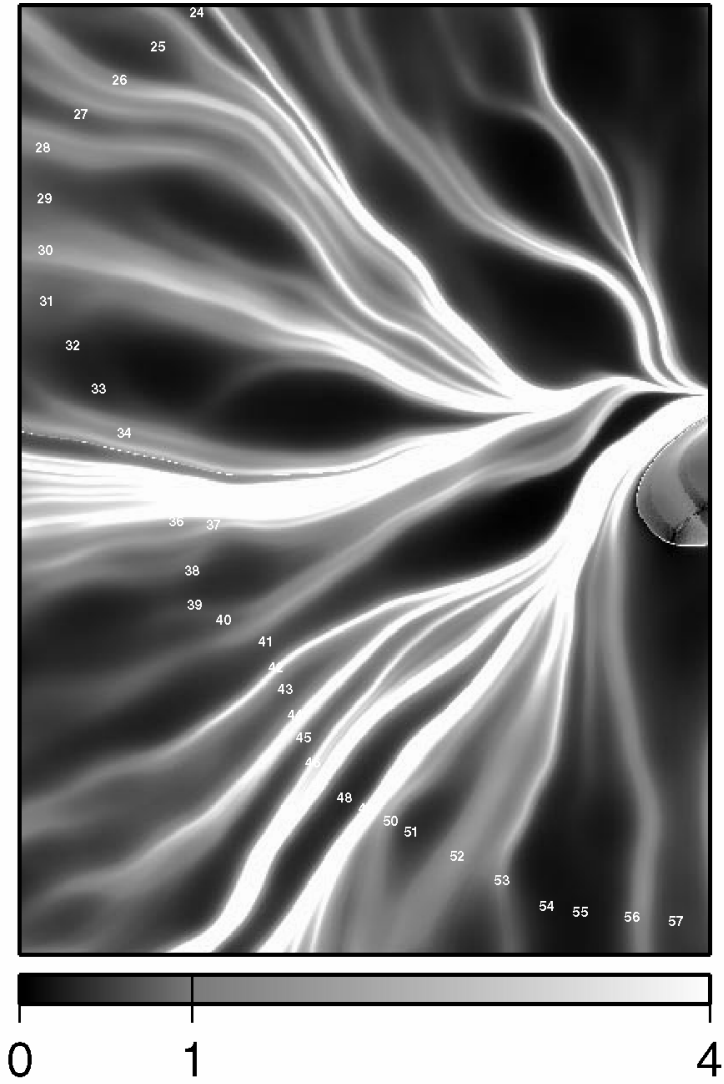

\section{F index}

Fig. 3. The map represents the Findex computed in the $L G B$ region using the Liu and others (1999) $1 \mathrm{~km} D E M$ with $L=$ $50 \mathrm{~km}$.

sions of the DEM. We applied the BW method on each of these versions to produce 21 different velocity maps. The next step was to digitize coordinates (2161 values), based on the LGB traverse (see section 4), to produce a $2000 \mathrm{~km}$ profile around the LGB with approximately a point every $1 \mathrm{~km}$. At each of these points and for each smoothed version of the DEM, the balance velocities were computed using the FL method, and the BW velocities were interpolated at these points from the corresponding velocity map.

Along this profile we have defined three different zones: zone 1 corresponds to the first $925 \mathrm{~km}$ of the profile (the western part of the LGB); zone 2 corresponds to the glacier inflow region, including Mellor and Lambert Glaciers; and zone 3 corresponds to the other end of the profile (the eastern part of the LGB; see Fig. 2a). For each smoothing distance and for each zone, the mean differences and the rms between balance velocities obtained with the two methods $\left(U_{\mathrm{bFL}}-U_{\mathrm{bBW}}\right)$ were computed. Moreover, in order to see the convergence of each method, the mean difference and rms were also computed between the velocity profile obtained with a smoothed version of the DEM and the velocity profile obtained from the previous smoothed version (i.e. $\operatorname{DEM}(i)-\operatorname{DEM}(i-1)$, where $i=1,21$ ). One can see in Figure 1 that, in terms of the mean difference and rms, the FL method (grey line) and the BW method (dashed grey line) converge towards a stable solution as the smoothing distance increases. The BW scheme is, however, found to be much less sensitive to the smoothing distance than is the FL scheme. Indeed, in low-velocity regions (i.e. zones 1 and 3), until a smoothing distance of at least $50 \mathrm{~km}$ is reached, the
FL method has much higher variability than the BW method. In high-velocity regions (i.e. zone 2), if one uses the FL method, the DEM needs to be smoothed over distances of $100 \mathrm{~km}$ or more to reproduce a variability comparable to the BW method. In Figure 2 we have represented the computed balance velocities along the whole profile for two selected smoothing length scales $(L=50$ and $L=100 \mathrm{~km})$. We can see in this figure that the FL scheme produces, at short smoothing length scales and in particular glacier regions, narrow and high-intensity peaks. These peaks are not computing artefacts, they denote the tendency of the FL scheme to over-concentrate the flow at the centre of topographic depressions. We defined the quantity

$$
F=\frac{\int_{0}^{x} l(x) \mathrm{d} x}{l_{0} x},
$$

where $x$ is the curvilinear distance from the flow source $(x=0)$ and $l$ is the distance between two adjacent flowlines. This quantity is the ratio between the drainage surface of the ice sheet and the surface drained by the equivalent parallel flow and can be seen as the relative state of convergence or divergence of the topography. If we map this quantity in the LGB region, using the $1 \mathrm{~km}$ version of the Liu and others (1999) DEM (filtered at a $50 \mathrm{~km}$ smoothing scale) and using the FL scheme, we can observe that this scheme produces a very coherent pattern of highly convergent, narrow channel flow that obviously represents a signature of the topography (see Fig. 3). This signature cannot be directly interpreted as a velocity map but it is most probably linked to actual or past flow conditions.

\subsection{The role of the grid size}

As the BW scheme is dependent on the chosen grid size, the two methods are not exactly comparable. Indeed the grid size in the BW method plays the role of an additional smoothing parameter compared with the FL method, which is less sensitive to it. We have computed the two balance velocities along the LGB traverse profile using the $1 \mathrm{~km}$ DEM. Figure 2c shows that, with this grid size, the BW velocity contains more high-frequency information and is now much closer to the FL velocity. This is confirmed by the mean difference and rms value (see star in Fig. 1). Thus, comparison between the two methods shows that the FL scheme is very sensitive to the smoothing filter applied to the DEM, and indeed needs a large amount of smoothing to be comparable to the BW scheme. On the other hand, the BW scheme is sensitive to the size of the gridcell.

\section{COMPARISON WITH GPS DATA}

Between 1990 and 1995, Australian scientists carried out a series of glaciological traverses around the LGB. This traverse route, between the Mawson and Davis stations, approximately followed the $2500 \mathrm{~m}$ surface elevation contour, and data were collected at 73 ice-movement stations spaced approximately $30 \mathrm{~km}$ apart. Recently, Manson and others (2000) have processed the GPS data collected at these 73 stations along the LGB traverse route to produce precise surface velocities. Before the GPS velocities can be compared with the balance-velocity results, the GPS surface values must be converted to depth-averaged velocities. The ratio between surface speed and depth-averaged speed 
Smoothing length $=100 \mathrm{~km} \&$ Gridsize $=5 \mathrm{~km}$

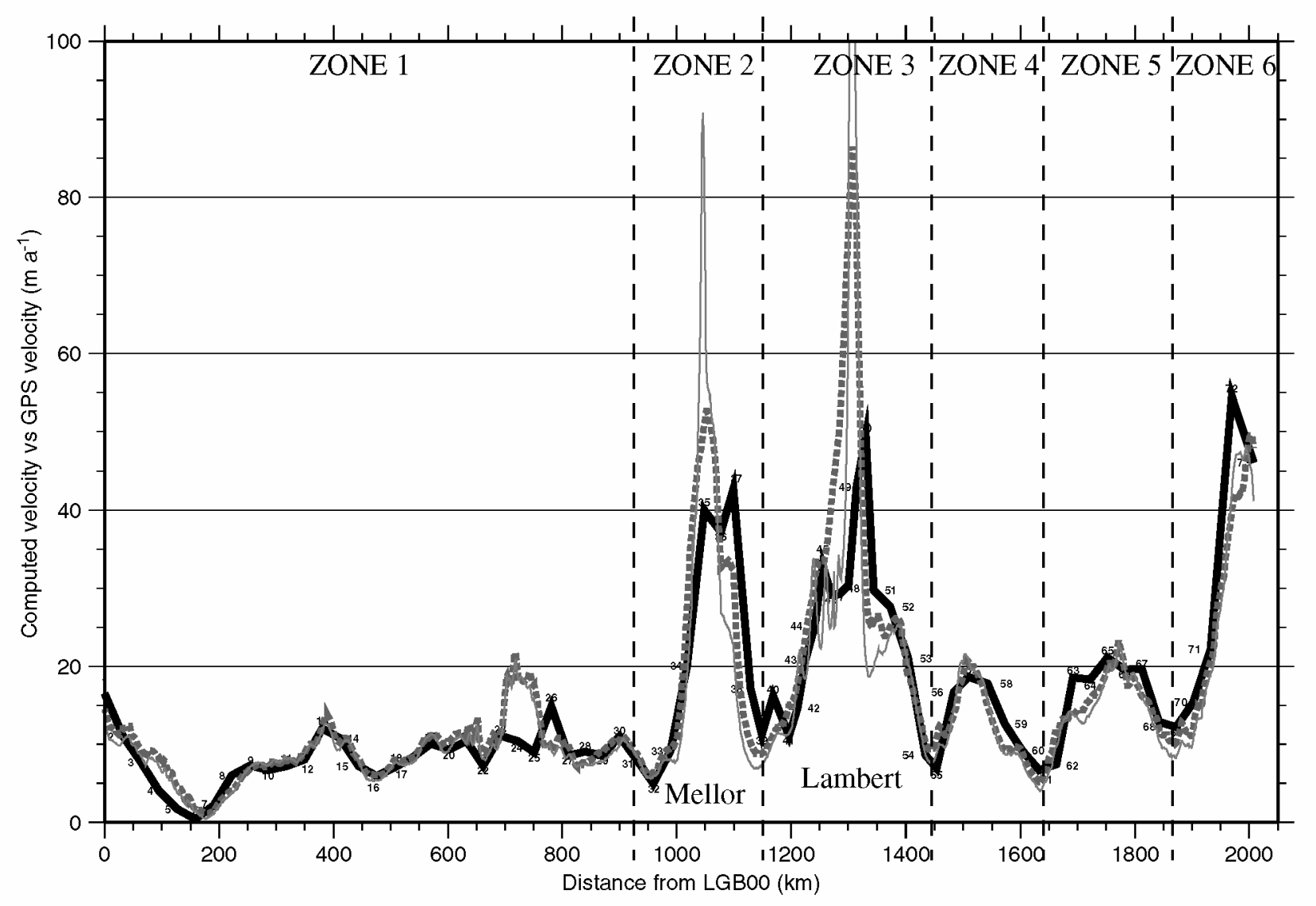

Fig. 4. Balance velocities around the LGB traverse route. The solid black line is the GPS velocity, the dashed grey line the BW computed velocity and the solid grey line the FL velocity. The numbers along the black line are the LGB stations, starting from the western side of the basin and finishing on the eastern side.

depends on ice rheology and the physical conditions in the ice column and at the base of the ice layer. A ratio of 0.87 has been applied between depth-averaged and surface velocity values, as defined in Budd and Warner (1996).

Figure 4 presents the comparison between the 73 GPS velocity measurements (black line) and the 2161 computed velocity points along the LGB (grey lines) using the $5 \mathrm{~km}$ DEM (Rémy and others, 1999) with $L=100 \mathrm{~km}$. Figure 4 shows that both computed balance velocities are in good agreement with the GPS-derived estimates. However, a closer look at Figure 4 also reveals that significant differences still exist, especially in the high-flow glacier regions, such as Mellor and Lambert Glaciers. In order to assess the capability of both methods to reproduce the intensity along the LGB traverse route, we have defined six separate zones (see Fig. 4). In each of these zones, the mean relative difference between modelled and observed velocity was computed as a function of the smoothing length scale (see Fig. 5). We adopted the convention that all percentages shown in parentheses below correspond to the FL method.

For the whole profile, the mean computed balance velocity is underestimated by about $9 \%(15 \%)$ compared with the mean GPS velocity. In low-motion regions (zones 1, 4 and 5) we can see (Figs 4 and 5) that the balance velocities are quite well modelled: the computed velocities in the western part of the LGB (zone 1) are higher by $\sim 10 \%$ (2$3 \%$ ) when compared with the GPS values, and in the eastern part (zones 4 and 5) the values are generally smaller by $\sim 10 \%(12 \%)$ and $\sim 5 \%(10 \%)$ respectively. These percentages are within the input data uncertainties and method- ology, but a small imbalance between these two sides of the LGB could also explain these differences. The high-velocity regions (zones 2,3 and 6) are found to have higher computed balance velocities of about 25\% (15\%) for Lambert Glacier, near-identical velocities 3\% (-3\%) for Mellor Glacier and smaller velocities of about $18 \%(-20 \%)$ for the eastern part. A part of this difference in velocities within the highervelocity regions could be due to ice-sheet sliding. Indeed, when sliding is assumed for velocities $>30 \mathrm{ma}^{-1}$ (i.e. for these velocities, the ratio between depth-averaged and surface velocity is taken to be 1 instead of 0.87 ), the relative difference between computed and measured velocities decreases to $10 \%(1 \%)$ for Lambert Glacier (but increases for zones 2 and 6). The spatial sampling of the LGB stations (i.e. distances of about $30 \mathrm{~km}$ between stations) could also miss some highvelocity, smaller-scale streams, which would lead to an underestimation of the mean GPS velocity.

We can see from these comparisons that the methodology adopted for the balance-velocity computation accounts for 5$10 \%$ of the error in the final velocity. We review other possible sources of uncertainty in the next section.

\section{OTHER SOURGES OF UNGERTAINTY}

\section{Ice thickness}

In the mass-balance equation (Equation (1)), the relative error in balance velocity due to an error in the local icethickness value is simply equal to the relative error in the ice thickness. This error in relative ice thickness can reach 

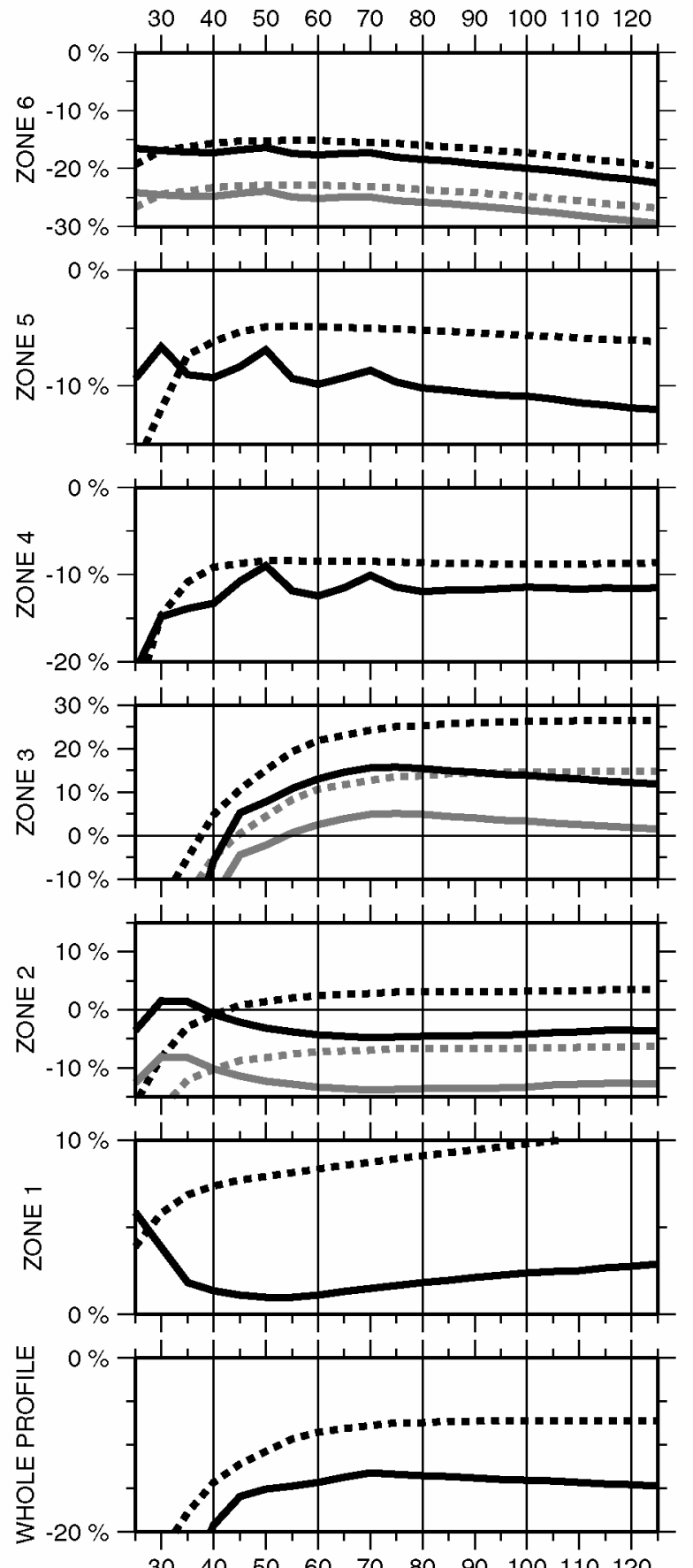

$\begin{array}{llllllllll}30 & 40 & 50 & 60 & 70 & 80 & 90 & 100 & 110 & 120\end{array}$

Smoothing length $(\mathrm{km})$

Fig. 5. The relative difference (\%) between computed and $G P S$ velocities for the whole LGB traverse profile and for each of the six zones defined in Figure 4. The solid black line is the $F L$ velocity, and the dashed black line the $B W$ velocity. The grey lines are the corresponding relative differences when sliding is considered $\left(\right.$ ratio $=1$ for $\left.V_{\mathrm{gps}}>30 \mathrm{ma}^{-1}\right)$.

a few tens of per cent in regions where data are sparse. In the LGB region, many ice-thickness measurements have been collected, and these data are included in the BEDMAP compilation. Therefore along the LGB traverse, the relative ice-thickness error is estimated to be only a few per cent, leading to an equivalent few per cent error in the computed balance velocity.

\section{Accumulation}

The accumulation is an integrated quantity, and hence a random fluctuation in the accumulation rate will not have a great impact on the balance velocity. A fluctuation of about $10 \%$ in the accumulation rate randomly distributed on the whole ice sheet and decorrelated at the scale of the grid size $(5 \mathrm{~km})$ leads to relative variation in the computed velocity of only a few per cent. On the other hand, a bias in the accumulation at a regional scale will have a strong effect in terms of the computed velocity magnitude. The use of two different accumulation datasets (e.g. Radok and others, 1986; Vaughan and others, 1999), which show strong regional-scale differences, can lead to differences of $>50 \%$ in the computed magnitude of the velocity.

At the LGB stations, we have performed a series of balance-velocity computations using the four "best"-performing accumulation datasets identified by Fricker and others (2000) in this region: (i) the Australian Antarctic CRC dataset (Budd and Smith, 1982); (ii) the Global Atmospheric Assimilation and Prediction Scheme (GASP) (Budd and others, 1995); (iii) the Commonwealth Scientific and Industrial Research Organisation (CSIRO) dataset (Smith and others, 1998); and (iv) the Vaughan dataset (Vaughan and others, 1999). The CRC and Vaughan datasets are based on field observations, whilst the GASP and CSIRO datasets were determined from atmospheric models. The results of these comparisons show that using accumulation values from an atmospheric model, as opposed to an observational dataset, produces velocity differences of around $30 \%$, with significantly higher percentage differences at a number of sites (see Hurd, 2002). In general, the choice of accumulation input datasets caused the computed velocities to vary from the GPS-derived values by up to $55 \%$.

\section{Surface topography}

Two different altimeter-derived DEMs (Liu and others, 1999; Rémy and others, 1999) have been used to test balance-velocity results in the LGB region. The computation of the $1 \mathrm{~km}$ velocities along the LGB traverse using these two different DEMs shows, at equal smoothing, near-identical results in terms of velocity magnitude but exhibits small distortions in spatial geometry. Cross-correlation of the computed velocities shows generally very high correlation ( $>0.92$ ), but also indicates some lags of a few km, mostly in regions of high convergence-divergence. These lags are due to geometrical differences in the surface topographies of the two DEMs. These distortions are of the same magnitude as those that can be seen in Figure 4 between the GPS and the computed balance velocities (see, e.g., LGB stations 6 and 50). Indeed, one can see that the maximum (minimum) computed velocity occurs a few $\mathrm{km}$ before (after) the maximum (minimum) GPS values. Hence these lags between observed and modelled velocity can probably be explained by the fact that the DEMs used here are not exactly identical as they do not match the true surface of the ice sheet. Additionally, the smoothing applied to the topography causes modification of the shape of the surface contours.

\section{GONGLUSION}

Comparison between computed balance velocities and GPS measurements, observed along the Australian LGB traverse route, shows that actual balance-velocity computing schemes are able to recover the spatial pattern of the velocities and to model their average intensity to within 5- 
$25 \%$. The major sources of error in this region, where ice thickness values have been collected and hence are assumed well known, are found to be the uncertainties present in the accumulation rate, the adopted computational scheme (either the FL or BW method) and the smoothing length scale $(L)$ applied to the topography. With improvement in the resolution of Antarctic DEMs likely from satellite missions such as ICESat, and an increasing refinement in the accumulation-rate and ice-thickness data, the dominant sources of these errors can be removed. Despite improvement in input data sources, the role of the filter used in the balance-velocity calculations still needs to be further investigated, especially aspects such as the type of filter used, the optimum smoothing distance, etc.

In this study, the BW method was found to be very robust, and with high-resolution DEMs (1 km grid size) the method was able to capture the high-flow regions of the glacier streams. The FL method was very sensitive to the adopted smoothing-length scale, but when the topography was sufficiently smoothed (at longer spatial scales), the resulting balance velocities were equivalent to the BW values.

\section{ACKNOWLEDGEMENTS}

The work by R. Hurd and R. Coleman was supported by grants from the Australian Research Council and the Australian Antarctic Science Grants Scheme. R. Hurd was supported by an Australian Postgraduate Award held at the University of Tasmania. The authors are grateful to the scientific editor and the two anonymous referees for their valuable comments which led to great improvements in this paper.

\section{REFERENGES}

Bamber, J. L. and P. Huybrechts. 1996. Geometric boundary conditions for modelling the velocity field of the Antarctic ice sheet. Ann. Glaciol., 23, 364-373.

Bamber, J. L., R.J. Hardy and I. Joughin. 2000a. An analysis of balance velocities over the Greenland ice sheet and comparison with synthetic aperture radar interferometry. F. Glaciol., 46(152), 67-74.

Bamber, J. L., D. G. Vaughan and I. Joughin. 2000b. Widespread complex flow in the interior of the Antarctic ice sheet. Science, 287(5456), 12481250 .

Budd, W. F. and I. N. Smith. 1982. Large-scale numerical modelling of the Antarctic ice sheet. Ann. Glaciol., 3, 42-49.
Budd, W. F. and R. C. Warner. 1996. A computer scheme for rapid calculations of balance-flux distributions. Ann. Glaciol., 23, 21-27.

Budd, W. F., P. A. Reid and L. J. Minty. 1995. Antarctic moisture flux and net accumulation from global atmospheric analyses. Ann. Glaciol., 21, 149-156.

Drewry, D. J., S. R. Jordan and E. Jankowski. 1982. Measured properties of the Antarctic ice sheet: surface configuration, ice thickness, volume and bedrock characteristics. Ann. Glaciol., 3, 83-91.

Fricker, H. A., R. C. Warner and I. Allison. 2000. Mass balance of the Lambert Glacier-Amery Ice Shelf system, East Antarctica: a comparison of computed balance fluxes and measured fluxes. F. Glaciol., 46(155), 561-570.

Hurd, R. 2002. Investigations of glaciological and meteorological parameters in the Lambert Glacier basin using high precision GPS techniques. (Ph.D. thesis, University of Tasmania.)

Joughin, I., M. Fahnestock, S. Ekholm and R. Kwok. 1997. Balance velocities of the Greenland ice sheet. Geophys. Res. Lett., 24(23), 3045-3048.

Joughin, I. and 7 others. 1999. Tributaries of West Antarctic ice streams revealed by RADARSAT interferometry. Science, 286(5438), 283-286.

Liu, H., K. C. Jezek and B. Li. 1999. Development of an Antarctic digital elevation model by integrating cartographic and remotely sensed data: a geographic information system based approach. 7. Geophys. Res., 104(B10), 23,199-23,213.

Lythe, M. B., D. G. Vaughan and BEDMAP Consortium. 2001. BEDMAP: a new ice thickness and subglacial topographic model of Antarctica. $\mathcal{f}$. Geophys. Res., 106(B6), 11,335-11,351.

Manson, R., R. Coleman, P. Morgan and M. King. 2000. Ice velocities of the Lambert Glacier from static GPS observations. Earth Planets Space, 52(11), 1031-1036

Morgan, V. I., T. H. Jacka, G. J. Akerman and A. L. Clarke. 1982. Outlet glacier and mass-budget studies in Enderby, Kemp and Mac. Robertson lands, Antarctica. Ann. Glaciol., 3, 204-210.

Paterson, W. S. B. 1994. The physics of glaciers. Third edition. Oxford, etc., Elsevier. Radok, U., R. G. Barry, D. Jenssen, R. A. Keen, G. N. Kiladis and B. McInnes. 1982. Climatic and physical characteristics of the Greenland ice sheet. Part I and II. Boulder, CO, University of Colorado. Cooperative Institute for Research in Environmental Sciences.

Radok, U., T.J. Brown, D. Jenssen, I. N. Smith and W. F. Budd. 1986. On the surging potential of polar ice streams. Part IV. Antarctic ice accumulation basins and their main discharge regions. Boulder, CO, University of Colorado. Cooperative Institute for Research in Environmental Sciences; Melbourne, University of Melbourne. Meteorology Department. (U.S. Department of Energy Report DE/ER/60197-5.)

Rémy, F. and J. F. Minster. 1993. Precise altimetric topography in ice-sheet flow studies. Ann. Glaciol., 17, 195-200.

Rémy, F., P. Shaeffer and B. Legrésy. 1999. Ice flow physical processes derived from ERS-1 high-resolution map of Antarctica and Greenland ice sheet. Geophys. 7. Int., 139(3), 645-656.

Smith, I. N., W. F. Budd and P. Reid. 1998. Model estimates of Antarctic accumulation rates and their relationship to temperature changes. Ann. Glaciol., 27, 246-250.

Vaughan, D. G., J. L. Bamber, M. B. Giovinetto, J. Russell and A.P.R. Cooper. 1999. Reassessment of net surface mass balance in Antarctica. 7. Climate, 12(4), 933-946. 